\title{
Binocular rivalry with moving patterns
}

\author{
NICHOLAS J. WADE \\ University of Dundee, Dundee, Scotland \\ CHARLES M. M. DE WEERT \\ University of Nijmegen, Nijmegen, The Netherlands \\ and \\ MICHAEL T. SWANSTON \\ Dundee College of Technology, Dundee, Scotland
}

\begin{abstract}
Binocular rivalry between a horizontal and a vertical grating was examined in six experiments. The gratings could be presented in a static form or dynamically so that either one or both gratings moved. The motion consisted of a symmetrical transformation of the gratings about their centers, so that the lines moved outwards or inwards. During rivalry, a moving pattern was visible for about $50 \%$ longer than an equivalently oriented static pattern (Experiments 1, 2, and 4). When both gratings were in motion (Experiments 3 and 5), the course of rivalry was similar to that found for two static gratings. The duration of dominance of the moving grating was influenced by its velocity (Experiment 6). The results are interpreted in terms of the stimulus strengths of the static and dynamic patterns.
\end{abstract}

The experimental examination of binocular rivalry has a long history. Du Tour (1760) described the fluctuations in visibility that followed viewing different colored papers before each eye, and Wheatstone (1838) demonstrated the rivalry that resulted from the presentation of grossly dissimilar contours in his stereoscope (see Helmholtz, 1925, for a summary of the early work). Subsequent research on binocular rivalry has been virtually confined to variations in the colors or patterns of the dichoptic stimuli. The particular variables manipulated in recent years have reflected the concern with identifying stimulus dimensions that might relate to the channels in vision isolated by neurophysiologists (Fahle, 1982; Wade, 1975a), and with the pattern characteristics that determine the stimulus strength of rivalrous pairs (Levelt, 1965; de Weert \& Levelt, 1974). Levelt defined this as being proportional to the amount of contour and contrast per unit area.

Relatively little experimental manipulation of moving patterns has been conducted, although this is known to be a salient feature for exciting neurons throughout the visual system. More pertinently, the conditions for which rivalry obtains under normal

The visits of N.J.W. to Nijmegen and C.M.M.W. to Dundee were supported by NATO Research Grant 093.82 . N.J.W. and M.T.S. were supported by the Medical Research Council of Great Britain (Grant G8104633N). N. J. Wade's permanent mailing address is: Department of Psychology, University of Dundee, Dundee DD1 4HN, Scotland. viewing conditions would inevitably involve motion. Breese (1899), who initiated the modern methods for measuring rivalry alternations, did report an experiment with moving gratings. He presented a stationary pattern of green and black diagonal lines to one eye, and moving red and black, oppositely oriented, diagonals to the other. The red grating was moved by means of a pendulum behind a fixed square aperture. "The moving lines of the red field were seen practically all the time. The stationary green field came and went with its usual regularity. The length of time it remained, however, was somewhat shorter than under normal conditions. While the green field was present the lines of the red field were still seen moving back and forth, seemingly through the lines of the grcen field" (Breese, 1899, pp. 30-31). Breese's concern with stimulus movement was in the context of the effects produced by eye movements, since he found that predominance (the total visibility time of a monocular stimulus) was increased for those patterns inducing most eye movements.

It is within the same context that some of the more recent studies on motion and rivalry have been conducted. Enoksson $(1961,1963,1968)$ has examined the consequences of motion in opposite directions on both rivalry and optokinetic nystagmus. For example, a vertical grating was moved leftwards in one eye and rightwards in the other. This induced optokinetic nystagmus, with its slow phase alternating in direction. The direction of motion seen in rivalry generally corresponded to that of the slow phase of 
nystagmus. This relationship has been investigated more intensively by Fox, Todd, and Bettinger (1975). They found a near perfect correlation between the direction of the slow phase of nystagmus and the direction of motion visible: when nystagmus direction changed so did the direction of phenomenal motion.

These results are clearly of importance in providing an objective indicator for binocular rivalry alternations, but they say less about the effects of motion on rivalry itself. For this, it is desirable to adopt a method more closely akin to Breese's, in which motion is present in one field alone. Fox and his colleagues have adopted this procedure in some studies on the selectivity of the rivalry suppression mechanism. For example, Fox and Check (1968) presented a large moving pattern to one eye in order to suppress a small, peripheral stationary target in the other. The static target was found to alternate in visibility in an abrupt manner "resembling an intermittent physical presentation" (p. 390). Similarly, Blake and Fox (1974) used a phase-alternating horizontal grating in one eye in order to enhance the suppression of a vertical grating in the other. Again they reported that rivalry was clear cut under these circumstances, which would not have been anticipated on the basis of Breese's description given above.

There are also several informal reports of the potency of motion in one eye to suppress the visibility of a pattern in the other. Springbett (1961) described the observations of 15 subjects who viewed a stationary pattern in one eye while a strip of white cardboard was moved in front of the other. The moving contour tended to suppress the visibility of the static pattern: "The effect, generally, is one of erasure, in that the part of the contralateral structure ahead of the movement remains intact while the part behind is obliterated"' (p. 106). Duensing and Miller (1979) used the same term, erasure, in their independent discovery of the same phenomenon. However, they also found that the erasure could operate over large and complex patterns (like the human face), and that fixated features of the static structure (like the eyes or lips) could retain their visibility despite motion of an arm over the contralateral visual field. Grindley and Townsend $(1965,1966)$ conducted a series of more systematic experiments to examine the effects of motion suppression. They presented a peripheral black square to the left eye, and could move a metal arm over the corresponding region of the right eye. During such movement of the arm, the stationary square often disappeared. Although the movement of the left eye was monitored during these experiments, the resolution was not sufficient to exclude eye movements as a factor involved in the disappearances. Among the many incidental observations contained in these papers, the following is of particular interest:
"If one screen was moved gently through about .5 to 1 deg this almost always led to temporary but complete obliteration of the objects on the screen" (Grindley \& Townsend, 1965, p. 101).

It is evident from all the studies described above that the stimulus movement employed would have initiated eye movements. That is, the movement presented to an eye was unidirectional and the normal response would have been to track it. The likelihood of eye tracking can be reduced by using moving displays that are transformed symmetrically from their centers, and it is with such displays that the present investigation is concerned. The stimuli in all cases were gratings, oriented vertically or horizontally, one to each eye. Motion could be imparted to either one or both gratings, and this consisted of symmetrical movement towards or away from their centers.

The first three experiments involved the cyclical expansion and contraction of the gratings, so that they underwent a spatial frequency change together with the outward and inward motion. These displays were produced by a video camera zooming towards or away from a stationary grating. Although this produced radial expansions and contractions of the gratings, the absence of any texture within the lines resulted in unambiguous perception of motion at right angles to the line orientation (see Swanston \& Wade, 1981a). The last three experiments utilized computer-generated gratings that could be moved towards or away from their centers without any spatial frequency change (apart from along the central strip). For each set of three experiments, two involved movement in one eye alone and the third consisted of motion in opposite senses (i.e., inward versus outward) in each eye.

\section{EXPERIMENT 1}

This experiment was designed to examine binocular rivalry with a stationary pattern in one eye and a moving pattern in the other. The stimuli consisted of vertical and horizontal gratings, each presented on a video monitor and combined binocularly by means of a modified prism stereoscope. The monitors received the outputs from two video cameras with motorized zoom lenses that were controlled by a microcomputer. The motion consisted of repetitive cycles of zoom-in (optical expansion) and zoom-out (contraction). Such expansion and contraction involved spatial frequency changes of about an octave. Accordingly, in different trials the stationary grating was presented at two spatial frequencies, corresponding to the highest and lowest spatial frequencies of the moving pattern. In addition, rivalry between the stationary gratings at the same or different spatial frequencies was also examined. 
Binocular rivalry typically involves periods in which either monocular pattern is phenomenally visible in its entirety, and also periods in which composites from the two fields are apparent (see Hollins, 1980; Wade, 1974). Preliminary observations confirmed that this occurred with the moving patterns, too. However, we could not support the generality of Breese's observation that the moving pattern appeared to be continuously present; it underwent phases of rivalry like that for the paired stationary patterns. In all the experiments, only those periods when either monocular stimulus was visible (i.e., either a vertical or horizontal grating alone) were reported by switch depressions; neither switch was depressed when composites were visible. The duration of composite visibility could be determined from the two predominance times and the trial time, although there are known to be problems associated with such derivations (see Swanston \& Wade, 1981b).

\section{Method}

Apparatus. The apparatus was an extension of the video system described by Swanston and Wade (1981a). It consisted of two video cameras (Sony AVC $3250 \mathrm{CE}$ ), each with a motorized zoom lens (Fujinon H $5 \times 14$ MA-1) under independent microcomputer control. The centers of two square-wave gratings were aligned with the optical axes of the lenses. The orientations of the gratings could be adjusted by the experimenter. The output of each camera was relayed to separate monitors (Hitachi VM $900 \mathrm{E} / \mathrm{K}$ ) situated in a darkened room. The face of each monitor was masked to provide a circular aperture subtending 6 deg at the subject's eyes. At maximum zoom-out, the spatial frequency of the grating on the monitor was $5.3 \mathrm{cycles} / \mathrm{deg}$, and at maximum zoom-in, it was $2.7 \mathrm{cycles} / \mathrm{deg}$. The zoom duration was $2 \mathrm{sec}$. The Michelson contrast of the gratings on the monitor was 0.7 , and the space averaged luminance on the screen was $2.6 \mathrm{~cd} / \mathrm{m}^{2}$.

The subject viewed the monitors, situated $114 \mathrm{~cm}$ from the eyes, through bi-prisms that could be adjusted to bring the circular apertures into alignment. A chinrest and forehead support stabilized the subject's head position. Two microswitches were located on a table in front of the subject, and these activated timers and recorders.

Subjects. The three authors acted as subjects. C.W. and N.W. were emmetropic, and M.S. wore an optical correction.

Design. The stimulus combinations always consisted of a vertical grating in one eye and a horizontal grating in the other, the presentations of which were balanced over eyes. A simplified representation of one of the combinations is shown in Figure 1. There were four combinations of rivalry for stationary patterns, produced by presenting the higher $(H, 5.3$ cycles/deg) or lower $(L$, 2.7 cycles/deg) spatial frequency grating to the left or right eye (i.e., H-H, H-L, L-H, and L-L). Similarly, there were four combinations involving movement: the zooming (Z) pattern in the left or right eye, with the higher or lower stationary spatial frequency grating in the other (i.e., Z-H, Z-L, L-Z, and H-Z). The order of the combinations was randomized within a session.

Procedure. The subject's task involved pressing the right-hand switch whenever the vertical grating alone was visible and the lefthand switch whenever the horizontal grating was completely visible. When composites were visible, neither switch was depressed. These responses were made for both the stationary and moving gratings. That is, the right-hand switch was depressed if a stationary vertical grating was visible, in some conditions, and when a moving vertical grating was visible, in others. Each trial lasted $60 \mathrm{sec}$. The moving gratings underwent cycles of zoom-in and zoom-out throughout this period, with a complete cycle lasting $4 \mathrm{sec}$.

A session consisted of 16 trials, one under each of the experimental conditions described above, then repeated with the vertical and horizontal gratings presented to the other eye. Each subject was tested in 10 sessions.

Index of rivalry dominance. The subjects responded to periods of complete dominance of the vertical or horizontal grating. Our interest was primarily in the influence of a moving pattern on its total duration of visibility (predominance time) when in rivalry with a stationary grating. To this end, we devised an index of rivalry dominance, called the predominance ratio (PR), that incorporated the predominance time for each target $\left(T_{a}\right.$ and $\left.T_{b}\right)$ :

$$
P R=\left(T_{a}-T_{b}\right) /\left(T_{a}+T_{b}\right)
$$

That is, the predominance ratio is the difference between the predominance times divided by their sum; $a$ and $b$ can refer to the eyes stimulated or to the patterns presented. That is, the predominance times can be partitioned in a variety of ways using this index; by averaging over the appropriate conditions, the influences of eye or pattern characteristics can be assessed. If the predominance times are equal, then $P R=0$ and the extreme values are -1.0 and +1.0 . Blake, Westendorf, and Overton (1980) and Fahle (1982) have used a similar predominance ratio, but in each case it was in terms of the predominance for one stimulus divided by that for the other. Such a ratio gives a value of 1.0 for equal predominances, but the extreme values are 0 and infinity. In order to compare values below and above 1.0, a reciprocal transformation on one of them is necessary. It is suggested that the index proposed here provides a more convenient and accessible means for comparing values when variations in predominance time accompany stimulus manipulations.

In order to facilitate interpreting the values given in the graphs below, the percentage predominance difference is determined by

Left Eye
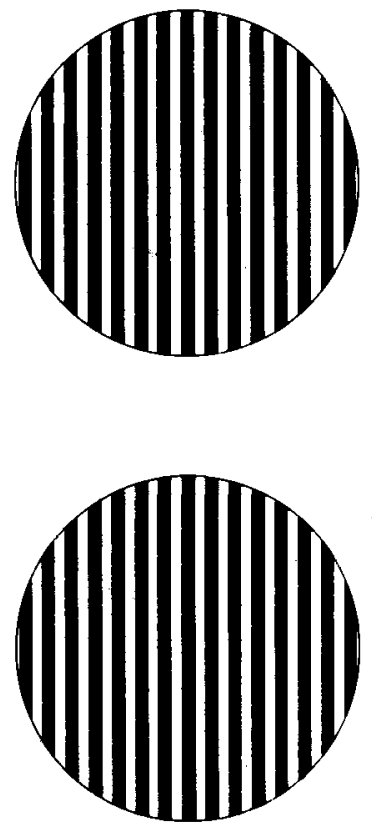

Figure 1. A schematic representation of one of the dynamic conditions tested in Experiments 1 and 2. The spatial frequency of the grating in the left eye remains constant while the zooming of the right eye pattern involves cyclical decresses and increases in spatial frequency over about 1 octave. 
the formula $[(1+P R) /(1-P R)-1)] \times 100$. Thus, the predominance times for one eye relative to the other are doubled or trebled for $P R=0.33$ and 0.50 , respectively.

\section{Results}

The moving gratings were not constantly visible, in the manner suggested by Breese (1899). Rather, they engaged in rivalry with a contralateral stationary pattern, so that subjects were readily able to report the orientation that was visible. The moving patterns were generally visible for a longer time than the stationary ones, although this varied with the spatial frequency of the latter. A common pattern of visibility during a cycle of zooming was that the stationary pattern dominated when the moving pattern was about the same spatial frequency. The PRs for the dynamic conditions will be considered separately from those for the static ones.

Moving stimull. The PRs for all the dynamic conditions were determined from the index $\left(T_{m}-T_{s}\right)$ / $\left(T_{m}+T_{s}\right)$, where $m$ and $s$ refer to the moving and stationary stimuli, combined over eyes and orientations. The values for each subject are given in Figure 2, for each spatial frequency of the static grating. It is clear that the moving gratings strongly predominate over the static ones in rivalry with them. The overall mean $P R$ is 0.22; that is, the moving gratings were visible for about $55 \%$ longer than the stationary gratings. It is also evident that this suppressive effect was not the same for both static gratings: a low $(2.7$ cycles/ deg) spatial frequency grating was suppressed for less time by a moving grating than was a higher ( $5.3 \mathrm{cy}-$ cles/deg) frequency grating. That is, the lower spatial frequency grating has a greater suppressive effect than a higher one.

The predominance ratios are calculated from the predominance times alone, and therefore do not reflect any variations in the frequencies $(F)$ or the individual periods $(t=T / F)$ of visibility. Measures of stimulus strength have typically utilized the variations in dominance periods rather than predominance times (see Levelt, 1965). Accordingly, the dominance periods were calculated for all the conditions above, and the period ratios- $\left(t_{a}-t_{b}\right) /\left(t_{a}+t_{b}\right)$ -were computed. The graphs for the period ratios were essentially the same as those for the predominance ratios: the increased predominance times for the moving stimuli were matched by corresponding increases in the dominance periods for the eye presented with motion.

The data described above were averaged over eyes and orientations. However, it was evident from the raw data that there were visibility differences between the orientations of the gratings. Accordingly, the PR was calculated for $\left(T_{v}-T_{h}\right) /\left(T_{v}+T_{h}\right)$ and averaged over all the dynamic conditions. Its value was +0.02 , indicating a $4 \%$ longer visibility of the vertical grating.

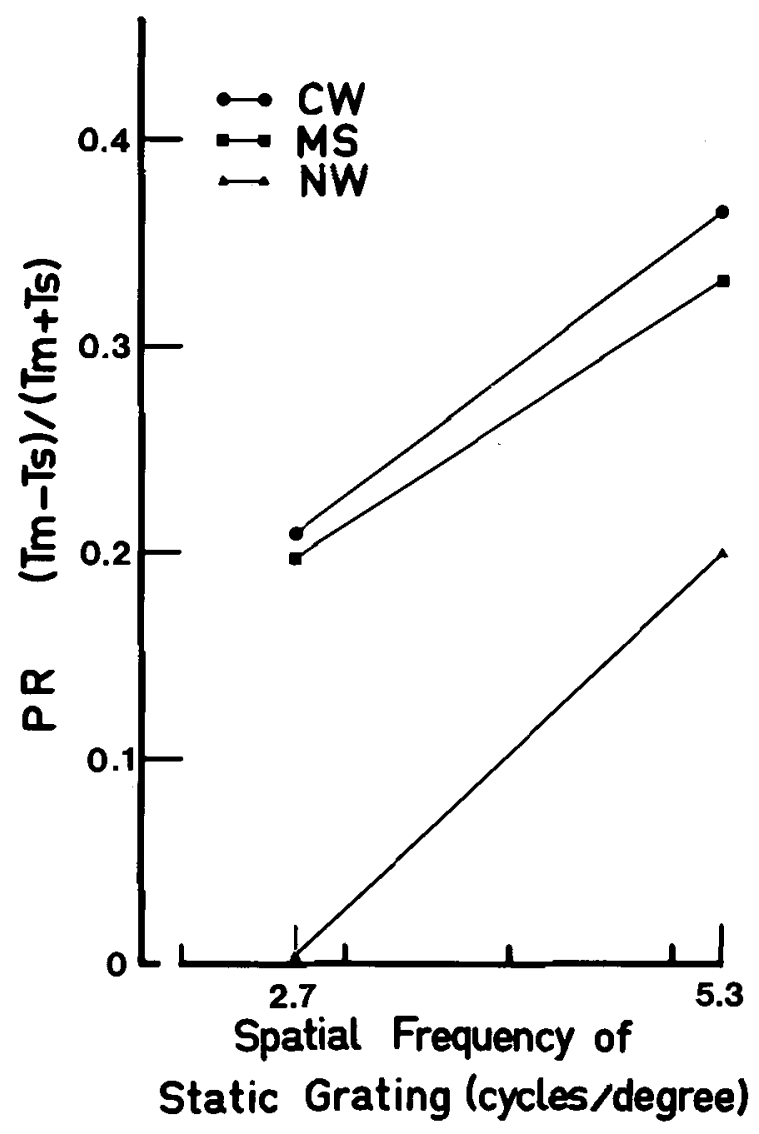

Figure 2. Predominance ratio (PR) of the moving pattern as a function of the spatial frequency of the static grating (Experment 1). The results for each subject are plotted separately.

Static stimuli. Similar effects can be seen in the data for rivalry between the static gratings. The PRs were computed for $\left(T_{1}-T_{r}\right) /\left(T_{1}+T_{r}\right)$, where 1 and $r$ refer to the stimuli in the left and right eyes. The values are given in Figure 3, averaged over grating orientations. With a dichoptic difference in spatial frequency, the eye receiving the lower one was relatively predominant. That is, the stimulus strength of the lower spatial frequency grating was greater than that of the higher spatial frequency pattern. When the spatial frequencies were the same in each eye, the PRs remained about the same, irrespective of the spatial frequency.

The same conclusions hold for dominance periods as for predominance times. The graphs for the period ratios correspond closely to those for PRs given in Figure 3. Thus, the lower spatial frequency gratings have greater stimulus strength, in terms of both predominance times and dominance periods, than the higher frequency grating in rivalry with it.

The three subjects showed the same effects, although the degree of lateral dominance between the eyes differed markedly. That is, with equivalent gratings in each eye, M.S. saw the one in the left eye for 


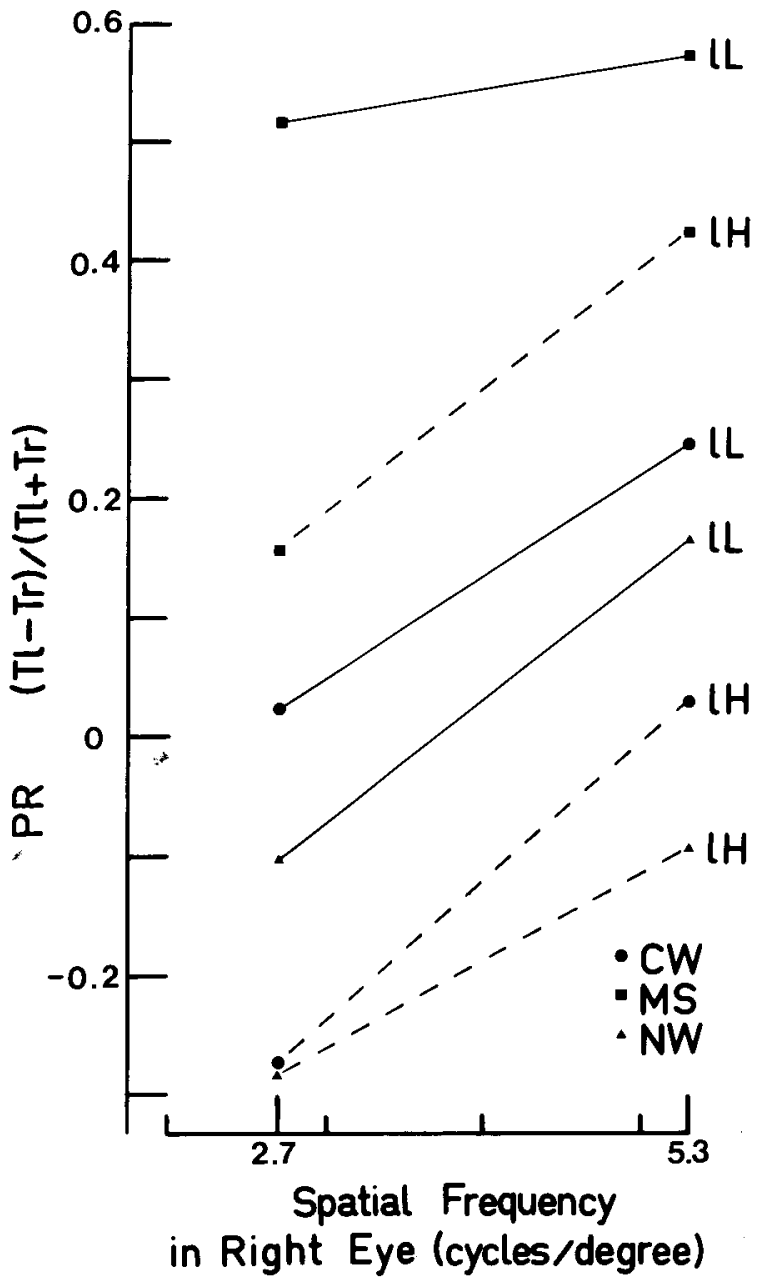

Figure 3. Predominance ratio (PR) as a function of the spatial frequency in the right eye for rivalry between stationary gratings (Experiment 1). The data for each subject are plotted for the lower and higher spatial frequencies in the left eye (IL and IH, respectively).

about three times as long as he saw that in the right; N.W. had a marginal right-eye rivalry dominance, and C.W. was slightly left-eye rivalry dominant. Nonetheless, each subject showed a similar effect of spatial frequency: a lower frequency grating was visible for longer than a higher frequency grating in rivalry with it.

These results correspond to those reported by Hollins (1980), who found what he called the peak effectiveness for binocular rivalry suppression at around 3 cycles $/ \mathrm{deg}$. Hollins suggested that the physiological mechanisms that respond to transient changes in stimulation play a role in rivalry, in addition to those that respond to pattern features. With static patterns, the transient system could be stimulated by pattern displacements consequent to involuntary eye movements. Kitterle and Thomas (1980) measured only the rate of rivalry and found that this reached a maximum at about 3 cycles $/ \mathrm{deg}$. Fahle (1982) used a different method for examining the influence of spatial frequency on rivalry. He presented single vertical and horizontal lines dichoptically, and then blurred the former systematically in order to remove the higher spatial frequency components: blurring resulted in a reduction in the duration of visibility of the vertical line. However, the comparison of the effects of such spatial filtering on a single line with the effects obtained in other experiments that employed gratings is not straightforward.

The effect of grating orientation was assessed as above and averaged over all the static conditions. Its value was +0.05 , indicating a $10 \%$ longer visibility of the vertical grating than of a horizontal one in rivalry with it. Wade (1974) found that a vertical grating was visible for about $18 \%$ longer than a horizontal grating in rivalry with it, and a similar effect was later found for single lines in rivalry (Wade, $1975 \mathrm{~b})$. These effects were not evident when the stimuli were viewed as afterimages. Kitterle and Thomas (1980) reported that the frequency of rivalry reversals increased with increasing orientation differences between gratings. Fahle (1982) also found a marked predominance of vertical over horizontal rivalrous lines.

Thus, movement in one eye is an effective stimulus for suppressing a stationary pattern in the other eye, but the suppression is by no means permanent, as Breese suggested. The spatial features of the stationary pattern also influence the course of rivalry.

\section{EXPERIMENT 2}

The fact that the higher frequency grating does not necessarily lead to higher predominance is of interest because of its possible relation to stimulus strength and rivalry. Considering stimulus strength as a function of spatial frequency and apparent contrast, it may be that the stimulus strength of the higher contrast grating is lower because it has a lower apparent contrast. For this reason, we lowered the whole range of spatial frequencies to a range below the maximum of the contrast sensitivity function. In this experiment, gratings ranging in spatial frequency from 1.3 to 2.6 cycles/deg were employed. Three static frequencies were examined, two corresponding to the initial and final states of the zooming grating and the third at an intermediate value.

\section{Method}

Apparatus. The viewing distance to the monitor screens was reduced to $57 \mathrm{~cm}$, and the screens were masked to an angular subtense of $6.2 \mathrm{deg}$, which was approximately the same as that employed in Experiment 1. Three static gratings, of 2.6, 2.2, and 1.3 cycles/deg, were presented. The moving (cyclically zooming) grating varied between the two extreme values over the same durations as in Experiment 1. 


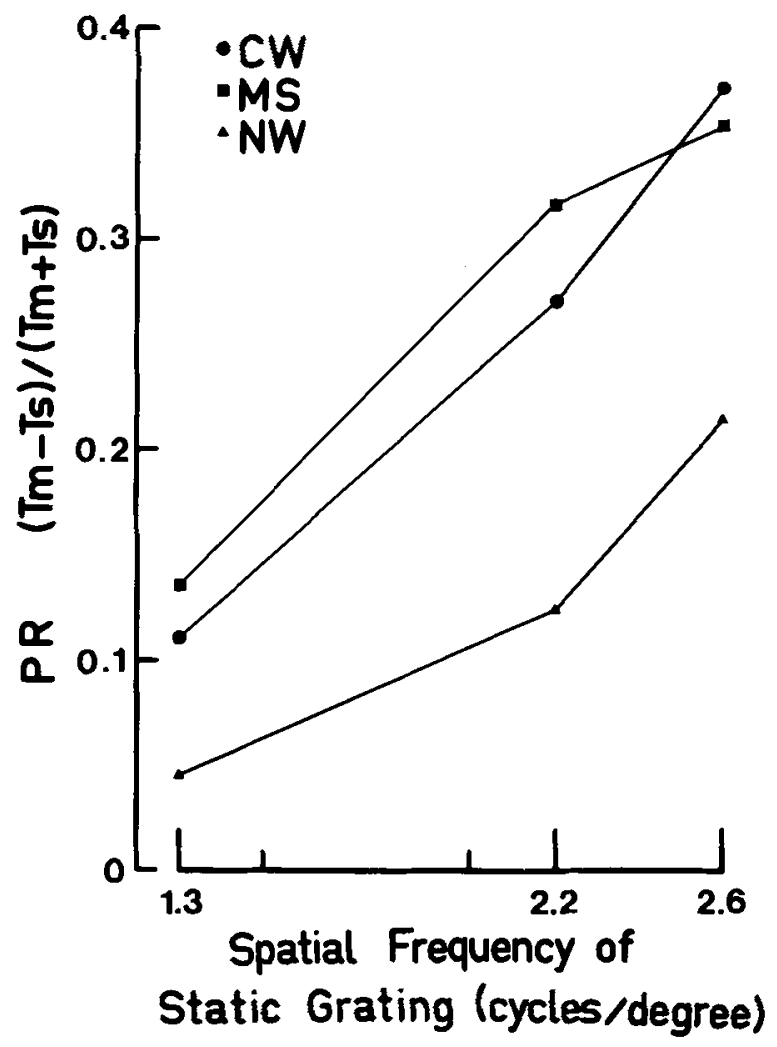

Figure 4. Predominance ratio (PR) of the moving pattern as a function of the spatial frequency of the static gnting (Experiment 2).

Subjects. The same three subjects participated in this experiment. Procedure. The general procedure was the same as that employed in Experiment 1. There were six rivalry combinations involving motion: zooming in the left eye with the static higher $(\mathrm{H})$, intermediate (M), and lower (L) spatial frequencies in the right, and vice versa. There were nine combinations of the static gratings, derived from all the possible combinations of the three spatial frequencies. A session consisted of a 60 -sec trial for each of the 15 conditions, presented in random order. Each subject was tested in 10 sessions, 5 with the vertical grating to the left eye and the horizontal to the right, and 5 with the alternative combination.

\section{Results}

The data provide general support for those found in Experiment 1. The moving pattern presented to one eye was visible for longer than any static grating in rivalry with it, and again the effect varied with the spatial frequency of the latter. The combinations involving the intermediate spatial frequency produced results between those at either of the extreme spatial frequency values.

Moving stimuli. For the moving pattern, the overall PR was the same as that found in Experiment 1, namely 0.22 , indicating that it was visible for about $55 \%$ longer than a stationary pattern in rivalry with it. The PRs for all the dynamic conditions are given in Figure 4 for each static spatial frequency. It will be noted that the range of spatial frequencies was lower than in the previous experiment and that the lowest value presented here $(1.3$ cycles $/ \mathrm{deg})$ had essentially the same suppressive effect as the lower one $(2.7 \mathrm{cy}$ cles/deg) employed in Experiment 1.

Once again, the graphs for the period ratios had the same form as those for the PRs shown in Figure 4. For C.W. and N.W., they were virtually equivalent, whereas for M.S. the slope was the same, but the period ratios were somewhat smaller in value than the PRs.

The effect of grating orientation on the PR was again determined for $\left(T_{v}-T_{h}\right) /\left(T_{v}+T_{h}\right)$; the value averaged over all dynamic conditions was +0.05 , indicating a $10 \%$ longer visibility for the vertical grating.

Static stimull. A similar pattern of results was found for rivalry between stationary gratings. The PRs for all static combinations are given in Figure 5.

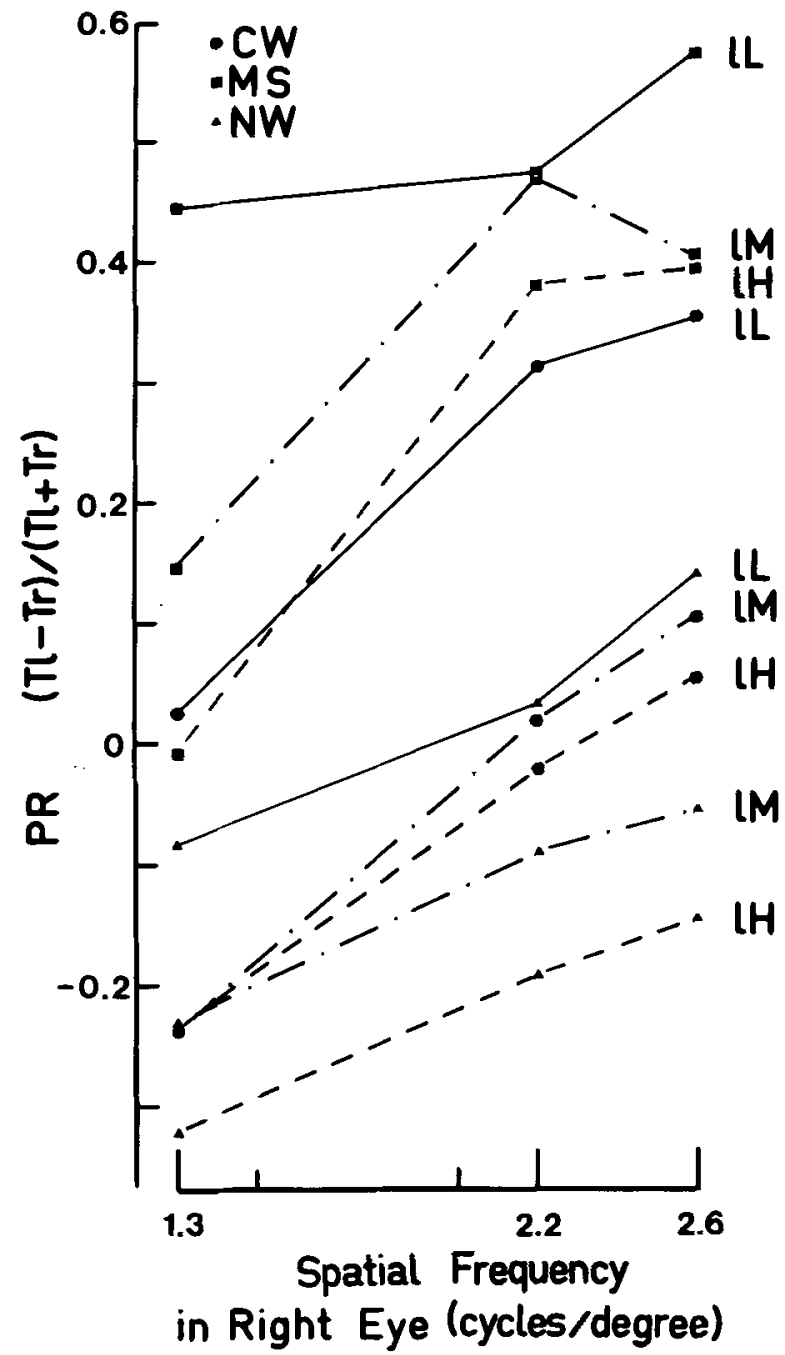

Figure 5. Predominance ratio (PR) as a function of the spatial frequency in the right eye for rivalry between two stationary gratings (Experiment 2). The data for each subject are plotted for the lower, medium, and higher spatial frequencies in the left eye (IL, IM, and IH, respectively). 
A grating of 1.3 cycles/deg had a greater suppressive effect than the higher frequency gratings in rivalry with it. Thus, once more, the lowest frequency grating yielded the greatest predominance. The same conclusions apply to the dominance periods: the individual periods of dominance were longer when the lower spatial frequency patterns were presented to an eye. It is tempting to conclude that stimulus strength in binocular rivalry does not correspond to the form of the contrast sensitivity function. A similar conclusion was reached by Hollins (1980), although his results as a whole differed from ours in the low spatial frequency range examined in this experiment. There were, however, certain differences in detail between the experiments reported by Hollins and ours. Hollins presented sinusoidal gratings within a 1-deg square field, whereas our displays were of square-wave gratings within a much larger (6-deg) circular field. It is possible that, in addition to the luminance profiles of the gratings, field size is an important factor in such experiments.

A stationary vertical grating was visible on average for about $15 \%$ longer than a horizontal grating $(P R=+0.07)$. This value is slightly greater than that found in Experiment 1, as was that for the dynamic stimuli. The difference was in the same direction for each subject. The similarity of the values for dynamic and static stimuli supports the view that the effects of orientation are not influenced by the motion of the gratings.

\section{EXPERIMENT 3}

A moving pattern suppresses a stationary pattern for longer than it is suppressed by one. Accordingly, it would be anticipated that the differential suppression attributed to motion would be nullified if it was presented dichoptically. This experiment examined rivalry with moving stimuli in both eyes. Vertical and horizontal gratings were again presented, but the optical expansion (zoom-in) and contraction (zoom-out) phases were either the same or different in the two eyes. That is, both gratings underwent changes in spatial frequency as a consequence of the zooming, but the transformations were either in-phase or outof-phase: both expanded and contracted together, or one expanded while the other contracted.

\section{Method}

Apparatus. The apparatus and viewing distance were the same as in Experiment 1.

Subjects. The same three subjects were tested.

Procedure. Four rivalry conditions were examined: in- or outof-phase motion with a horizontal grating to the left eye and a vertical grating to the right eye, and vice versa. The task was the same as in the previous experiments. The subjects reported, by switch depressions, whether the (moving) horizontal or vertical grating was visible in its entirety. Each combination was presented once within a session, and each subject was tested in 10 sessions.

\section{Results}

The phenomenal effects of the in- and out-of-phase rivalry conditions were reported to differ by all three subjects. The rivalry phases were shorter and more difficult to follow for the in-phase conditions. Moreover, subjects reported that the out-of-phase motion generally resulted in the dominance of whichever pattern was at the lower spatial frequency. That is, the spatial characteristics of the stationary gratings that were found to be of importance in the previous experiments also markedly influenced the course of rivalry between two moving patterns.

The predominance times were longer for rivalry with out-of-phase motion, and the dominance periods were longer (See Table 1). Moreover, composites occupied a larger proportion of the trial time for

Table 1

Predominance Times (in Seconds) and Response Frequencies for Visibility of the Left (l) and Right (r) Eye Patterns During Rivalry Between Gratings, Zooming Either In-Phase (IP) or Out-Of-Phase (OP)

\begin{tabular}{|c|c|c|c|c|c|c|c|}
\hline & & \multicolumn{2}{|c|}{ C.W. } & \multicolumn{2}{|c|}{ N.W. } & \multicolumn{2}{|c|}{ M.S. } \\
\hline & & Time & Frequency & Time & Frequency & Time & Frequency \\
\hline $\begin{array}{c}\mathbf{l H}-\mathrm{rV} \\
\mathrm{IP}\end{array}$ & $\begin{array}{l}1 \\
r \\
P R\end{array}$ & $\begin{array}{l}18.8 \\
18.2 \\
+0.02\end{array}$ & $\begin{array}{l}25.4 \\
26.5\end{array}$ & $\begin{array}{l}16.9 \\
23.6 \\
-0.16\end{array}$ & $\begin{array}{l}16.0 \\
21.5\end{array}$ & $\begin{array}{c}13.2 \\
5.9 \\
+0.39\end{array}$ & $\begin{array}{l}14.8 \\
11.2\end{array}$ \\
\hline$\underset{\text { IP }}{\text { IV }}$ & $\begin{array}{l}1 \\
r \\
P R\end{array}$ & $\begin{array}{l}22.6 \\
19.1 \\
+0.08\end{array}$ & $\begin{array}{l}25.9 \\
24.8\end{array}$ & $\begin{array}{l}17.7 \\
22.2 \\
-0.12\end{array}$ & $\begin{array}{l}15.8 \\
14.9\end{array}$ & $\begin{array}{c}18.6 \\
8.4 \\
+0.39\end{array}$ & $\begin{array}{l}20.2 \\
14.3\end{array}$ \\
\hline $\begin{array}{c}\mathrm{IH}-\mathrm{rV} \\
\mathrm{OP}\end{array}$ & $\begin{array}{l}1 \\
r \\
\text { PR }\end{array}$ & $\begin{array}{l}24.9 \\
23.7 \\
+0.02\end{array}$ & $\begin{array}{l}22.4 \\
20.7\end{array}$ & $\begin{array}{c}25.3 \\
30.9 \\
-0.10\end{array}$ & $\begin{array}{l}16.9 \\
17.1\end{array}$ & $\begin{array}{l}21.0 \\
10.5 \\
+0.35\end{array}$ & $\begin{array}{l}16.1 \\
14.3\end{array}$ \\
\hline $\begin{array}{c}\mathrm{IV}-\mathrm{rH} \\
\mathrm{OP}\end{array}$ & $\begin{array}{l}1 \\
r \\
\text { PR }\end{array}$ & $\begin{array}{r}25.1 \\
25.7 \\
0.00\end{array}$ & $\begin{array}{l}21.5 \\
24.8\end{array}$ & $\begin{array}{l}25.3 \\
29.6 \\
-0.08\end{array}$ & $\begin{array}{l}16.0 \\
16.0\end{array}$ & $\begin{array}{l}25.8 \\
10.3 \\
+0.44\end{array}$ & $\begin{array}{l}16.5 \\
14.9\end{array}$ \\
\hline
\end{tabular}

Note-The predominance ratios (PR) are calculated for $\left(T_{l}--T_{r}\right) /\left(T_{l}+T_{r}\right)$ for all conditions. (Experiment 3). 
in-phase transformations. The predominance ratios, however, did not differ systematically for the two motion conditions. The vertical gratings were visible for $4 \%$ longer than the horizontal gratings ( $P R=$ +0.02 ). This value is in close correspondence with those obtained in the dynamic conditions above.

These results would be predicted from those of the first two experiments. When the zooming was inphase, the spatial frequencies were always equivalent in each eye. The out-of-phase zooming would necessarily have involved spatial frequency differences between the eyes, and the lower spatial frequency gratings tended to dominate during motion as they have been found to do with stationary gratings. Since the lower spatial frequencies were present equally often in each eye and for each orientation, this would not be expected to yield any differences in the predominance ratios.

Thus, rivalry between two optically expanding and contracting gratings would seem to follow the same rules as those for paired stationary gratings with either the same or different spatial frequencies.

\section{EXPERIMENT 4}

The movement involved in the previous experiments was accompanied by spatial frequency changes. It is obviously desirable to investigate the effects of movement without such concomitant pattern changes, but this was not possible with the video equipment used. Accordingly, this issue was addressed using different apparatus, in which the stimuli were generated on a computer-driven television monitor. Vertical and horizontal gratings were presented on the same screen, and they were combined binocularly by means of a four-mirror stereoscope.

This experiment examined the effect of motion in one eye paired with a stationary grating in the other. The motion was produced by small discrete displacements of the lines, so that they appeared to move either outwards $(O)$ from the center or inwards (I) to the center. Except for the central strip in which lines were either being generated (for $O$ ) or being removed (for I), and at the extremities of the circular displays, the spatial frequency remained constant. Rivalry was measured with two stationary gratings as well as with one moving grating.

\section{Method}

Apparatus. A red-green-blue television monitor (Barco CTVM 5l/2) was used for presenting the stimuli, which were generated by a PDP-11/23 microcomputer (see Wittebrood, Wansink, \& de Weert, 1981, for a detailed description of the equipment). The vertical and horizontal gratings were presented on the same screen, and were combined binocularly by means of a four-mirror stereoscope. A septum maintained the separation of the two monocular stimuli. Either one or both gratings could be moved in discrete steps. The discreteness was due to the $50-\mathrm{Hz}$ frame rate. The cir- cular displays subtended $3.4 \mathrm{deg}$ at the eye. The Michelson contrast was 0.7 , and the space averaged luminance was $11 \mathrm{~cd} / \mathrm{m}^{2}$.

Due to display constraints, it was not possible to match the spatial frequencies of the vertical and horizontal gratings in either the static or moving conditions. The static spatial frequencies were: vertical, $5.4 \mathrm{cycles} / \mathrm{deg}$; horizontal, $5.0 \mathrm{cycles} / \mathrm{deg}$. The corresponding values for the moving gratings were: 4.5 and 6.0 cycles/ deg. For the same reasons, it was not possible to match the velocities of the vertical and horizontal gratings, which were 0.37 and $0.28 \mathrm{deg} / \mathrm{sec}$, respectively. However, it should be noted that the main purpose of this and the next two experiments was to examine the effects of constant inward or outward motion without any spatial frequency changes in a given condition.

Subjects. Three subjects were tested; two of them (C.W. and N.W.) had participated in the previous experiments. The third subject, K.S., wore an optical correction.

Procedure. Ten stimulus combinations were examined: stationary gratings to each eye (IS-rS); a stationary grating to the left eye and an inward motion to the right (IS-rI); a stationary grating to the left eye and outward motion to the right (IS-rO); and the equivalent conditions for the contralateral eyes (II-rS and 1O-rS). Each condition was measured with a horizontal grating in the left eye and a vertical grating in the right, and vice versa.

The task for the subject was the same as in the previous experiments: microswitches were depressed for the complete visibility of the horizontal grating (left switch) and the vertical grating (right switch). The trial duration was $60 \mathrm{sec}$, and each of the 10 conditions was measured within a session. C.W. and N.W. were tested in 10 sessions, and K.S. was tested in 5.

\section{Results}

The two subjects who had participated in the earlier experiments reported that the appearance of rivalry was very similar with both experimental arrangements. This is supported by the data for predominance times, response frequencies, and predominance ratios, which are given in Table 2. The PRs for the stationary condition were calculated for $\left(T_{1}-T_{r}\right) /\left(T_{1}+T_{r}\right)$, and they indicate a marginal righteye superiority for $C . W$. and N.W. and a more marked left-eye rivalry dominance for K.S. The PRs for the conditions involving movement were calculated for $\left(T_{m}-T_{s}\right) /\left(T_{m}+T_{s}\right)$. These show that the moving stimuli predominated over the stationary ones in a manner equivalent to that found in the previous experiments. Indeed, the correspondence is very close, as the overall PR for moving with respect to static stimuli is 0.22 . That is, the moving stimuli were visible on average for $55 \%$ longer than the stationary gratings in rivalry with them, which is almost exactly the value determined for Experiments 1 and 2. As found in the previous experiments, the dominance periods varied in a manner similar to that of the predominance times.

The differences in spatial frequencies of the gratings make comparisons of the orientation effects more difficult. However, with two stationary gratings, the vertical was visible for around $4 \%$ longer than the horizontal, even though its spatial frequency was slightly higher. The spatial frequency differences were greater with the moving stimuli, and the vertical predominated on average for about $20 \%$ 
Table 2

Predominance Times (in Seconds) and Response Frequencies for each Subject Under Five Conditions in Experiment 4

\begin{tabular}{|c|c|c|c|c|c|c|c|}
\hline & & & W. & & & & \\
\hline & & Time & Frequency & Time & Frequency & Time & Frequency \\
\hline $1 S-r S$ & $\begin{array}{l}1 \\
\mathbf{r} \\
\text { PR }\end{array}$ & $\begin{array}{l}21.5 \\
22.9 \\
-0.04\end{array}$ & $\begin{array}{l}16.2 \\
17.0\end{array}$ & $\begin{array}{l}19.6 \\
21.8 \\
-0.05\end{array}$ & $\begin{array}{l}12.5 \\
12.3\end{array}$ & $\begin{array}{l}27.2 \\
21.3 \\
+0.12\end{array}$ & $\begin{array}{l}11.6 \\
10.8\end{array}$ \\
\hline IS-rI & $\begin{array}{l}1 \\
\text { r } \\
\text { PR }\end{array}$ & $\begin{array}{l}20.1 \\
24.2 \\
+0.18\end{array}$ & $\begin{array}{l}18.0 \\
15.4\end{array}$ & $\begin{array}{l}12.8 \\
23.9 \\
+0.20\end{array}$ & $\begin{array}{l}12.1 \\
11.4\end{array}$ & $\begin{array}{l}17.3 \\
30.9 \\
+0.29\end{array}$ & $\begin{array}{r}9.1 \\
10.6\end{array}$ \\
\hline $1 \mathrm{~S}-\mathrm{rO}$ & $\begin{array}{l}1 \\
\text { P } \\
\text { PR }\end{array}$ & $\begin{array}{c}18.1 \\
28.4 \\
+0.22\end{array}$ & $\begin{array}{l}16.9 \\
17.2\end{array}$ & $\begin{array}{l}15.2 \\
29.7 \\
+0.31\end{array}$ & $\begin{array}{l}12.7 \\
11.6\end{array}$ & $\begin{array}{l}17.7 \\
26.3 \\
+0.19\end{array}$ & $\begin{array}{l}9.1 \\
9.8\end{array}$ \\
\hline $1 \mathrm{I}-\mathrm{rS}$ & $\begin{array}{l}1 \\
r \\
\text { PR }\end{array}$ & $\begin{array}{l}26.6 \\
21.3 \\
+0.11\end{array}$ & $\begin{array}{l}14.8 \\
16.4\end{array}$ & $\begin{array}{l}23.4 \\
16.6 \\
+0.17\end{array}$ & $\begin{array}{l}12.6 \\
12.6\end{array}$ & $\begin{array}{l}28.5 \\
15.1 \\
+0.30\end{array}$ & $\begin{array}{l}9.0 \\
7.7\end{array}$ \\
\hline 10-rS & $\begin{array}{l}\text { l } \\
\text { r } \\
\text { PR }\end{array}$ & $\begin{array}{l}27.7 \\
19.8 \\
+0.16\end{array}$ & $\begin{array}{l}17.0 \\
17.1\end{array}$ & $\begin{array}{l}25.4 \\
15.2 \\
+0.26\end{array}$ & $\begin{array}{l}12.2 \\
11.8\end{array}$ & $\begin{array}{l}29.9 \\
17.1 \\
+0.27\end{array}$ & $\begin{array}{l}8.8 \\
8.7\end{array}$ \\
\hline
\end{tabular}

Note-The predominance ratios (PR) for the static condition (IS-rS) was calculated for $\left(T_{l}-T_{r}\right) /\left(T_{l}+T_{r}\right)$ and those for the inward (I) and outward $(O)$ movement conditions were $\left(T_{m}-T_{s}\right) /\left(T_{m}+T_{s}\right)$.

longer than the horizontal. However, it should be noted that the velocity of the moving vertical contours was higher than that for the moving horizontal contours.

\section{EXPERIMENT 5}

Dichoptic moving stimuli were presented in this experiment. That is, the displays presented to each eye were transformed over time, either both being directed in the same sense (I or O) or oppositely. Therefore, the conditions are similar to those for Experiment 3 , but in this instance no spatial frequency changes were involved.
Method

Apparatus and Subjects. The same apparatus was used as in Experiment 4 , and the same three subjects were tested.

Procedure. Four combinations of stimulus movement were presented: 1I-rI, 1I-rO, 10-rI, and 1O-rO. Each one was measured with balanced presentations of the horizontal and vertical gratings over eyes, making eight trials in all. The task was the same as in the previous experiments. Each subject was tested in five sessions.

\section{Results}

The PRs, calculated from $\left(T_{1}-T_{r}\right) /\left(T_{1}+T_{r}\right)$, for the four conditions are presented in Table 3, together with the predominance times and response frequencies. The PRs do not vary greatly between conditions, although the two subjects who showed a greater

Table 3

Predominance Times (in Seconds) and Response Frequencies for Each Subject Under the Four Conditions in Experiment 5

\begin{tabular}{|c|c|c|c|c|c|c|c|}
\hline & & \multicolumn{2}{|c|}{ C.W. } & \multicolumn{2}{|c|}{ N.w. } & \multicolumn{2}{|c|}{ K.S. } \\
\hline & & Time & Frequency & Time & Frequency & Time & Frequency \\
\hline II-II & $\begin{array}{l}1 \\
r \\
\text { PR }\end{array}$ & $\begin{array}{l}22.7 \\
22.0 \\
+0.01\end{array}$ & $\begin{array}{l}22.7 \\
21.0\end{array}$ & $\begin{array}{c}15.5 \\
19.0 \\
-0.09\end{array}$ & $\begin{array}{l}13.1 \\
14.9\end{array}$ & $\begin{array}{l}23.7 \\
20.9 \\
+0.06\end{array}$ & $\begin{array}{l}14.7 \\
13.5\end{array}$ \\
\hline II-rO & $\begin{array}{l}\mathbf{l} \\
\mathbf{r} \\
\mathbf{P R}\end{array}$ & $\begin{array}{l}21.6 \\
25.7 \\
-0.08\end{array}$ & $\begin{array}{l}23.6 \\
26.6\end{array}$ & $\begin{array}{l}13.0 \\
18.8 \\
-0.17\end{array}$ & $\begin{array}{l}11.2 \\
14.3\end{array}$ & $\begin{array}{l}22.7 \\
22.2 \\
+0.01\end{array}$ & $\begin{array}{l}14.8 \\
14.8\end{array}$ \\
\hline 10-rI & $\begin{array}{l}1 \\
\mathbf{r} \\
\text { PR }\end{array}$ & $\begin{array}{l}22.3 \\
21.4 \\
+0.02\end{array}$ & $\begin{array}{l}26.5 \\
24.1\end{array}$ & $\begin{array}{l}16.5 \\
15.4 \\
+0.04\end{array}$ & $\begin{array}{l}10.4 \\
15.2\end{array}$ & $\begin{array}{l}21.7 \\
23.5 \\
-0.03\end{array}$ & $\begin{array}{l}14.9 \\
17.0\end{array}$ \\
\hline 10-rO & $\begin{array}{l}1 \\
\mathbf{r} \\
\mathbf{P R}\end{array}$ & $\begin{array}{c}19.8 \\
22.4 \\
-0.07\end{array}$ & $\begin{array}{l}25.1 \\
27.4\end{array}$ & $\begin{array}{c}14.6 \\
15.8 \\
-0.03\end{array}$ & $\begin{array}{l}11.3 \\
15.7\end{array}$ & $\begin{array}{l}24.5 \\
25.0 \\
-0.01\end{array}$ & $\begin{array}{l}15.1 \\
16.3\end{array}$ \\
\hline
\end{tabular}

Note-Movement, either inward (I) or outward (O), was presented to each eye, and the predominance ratios (PR) were calculated for $\left(T_{l}-T_{r}\right) /\left(T_{l}+T_{r}\right)$. 
effect for outward moving stimuli in Experiment 4 also experienced greater predominance for outward motion when it was in rivalry with inwardly directed movement. The predominance times were similar for the four conditions, unlike those for Experiment 3 in which spatial frequency changes accompanied oppositely directed motion.

There was again a marked $(30 \%)$ predominance of the vertical over the horizontal grating, in this case when both of them were moving. These results are in accord with those of the earlier experiments in demonstrating that lower spatial frequency gratings will predominate over higher ones. However, the lower spatial frequency vertical grating also had a slightly higher rate of motion. In order to determine the influence of velocity on rivalry, a final experiment was performed.

\section{EXPERIMENT 6}

The final experiment was designed to determine whether the speed of movement influences the predominance of a moving over a stationary grating. With the computer-driven displays, the displacements of the contours are discrete, so that the motion perceived is apparent rather than real. The speed of apparent motion could be controlled by varying the interval between successive displacements. A range of intervals was examined, all of which produced clearly visible, smooth apparent movement.

\section{Method}

Apparatus. The apparatus was the same as that employed in the previous two experiments. The lines of the grating could be displaced successively by 1.0 minarc every $20 \mathrm{msec}$. The speed of movement was controlled by the number (n) of 20 -msec intervals between each displacement. For $n=1$, the speed corresponded to $0.83 \mathrm{deg} / \mathrm{sec}$. The intervals employed here were $40,60,80$, and $100 \mathrm{msec}$, corresponding to speeds of $0.41,0.28,0.21$, and $0.17 \mathrm{deg} / \mathrm{sec}$.

Subjects. The subjects were the same three used in the last two experiments.

Procedure. Rivalry was measured for vertical and horizontal gratings. The vertical grating was always stationary, but it was presented to the left or right eye in a balanced order. The horizontal grating was either stationary or moving at one of four speeds. The order of presentation of the 10 trials was randomized within a session, and the task was as in the previous experiments. C.W. and N.W. were tested in 10 sessions, and K.S. was tested in 5.

\section{Results}

The PRs for each condition were calculated for $\left(T_{h}-T_{v}\right) /\left(T_{h}+T_{v}\right)$. Since the vertical grating was always stationary, this ratio was equivalent to $\left(T_{m}-T_{s}\right) /\left(T_{m}+T_{s}\right)$ for all conditions other than that with the two stationary gratings. The PRs for each subject are shown in Figure 6, as a function of the speed of movement. C.W. showed a marked predominance for the vertical grating in the static condition, whereas the other two subjects gave approximately equal predominance for this condition. All

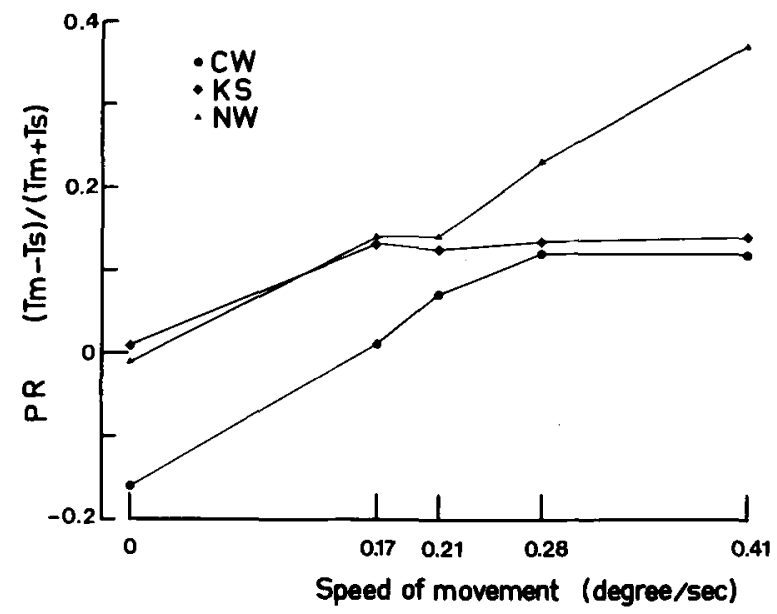

Figure 6. Predominance ratio (PR) as a function of the speed of the moving grating. The data for each subject are plotted separately.

subjects showed a marked predominance for the moving gratings. The overall PR for moving gratings was 0.14 ; that is, moving gratings were visible for $34 \%$ longer than stationary gratings in rivalry with them. While this value is lower than that found in Experiment 4 , this difference was probably influenced by the fact that the vertical grating was always stationary in the present experiment. The previous experiments had balanced orientations over eyes, but this was not done here because of the large number of trials that would have been involved. If the average PR of 0.14 is compared with that for the stationary gratings $(-0.05)$, then the moving horizontal gratings were visible for $\mathbf{4 4 \%}$ longer than the stationary horizontal grating in rivalry with a vertical one.

The dominance periods varied with velocity in a similar manner to the predominance times, and the curves for the period ratios follow closely those represented in Figure 6 for the PRs. Hence, as in all the other experiments reported, conclusions based on the PR values are consonant with those for the period measures that incorporate the frequencies of visibility.

The PRs for the moving gratings tended to increase with the velocity, although the curves varied considerably between subjects. Therefore, the orientation differences found in Experiments 4 and 5 in favor of the moving vertical grating are likely to have been exaggerated by its higher velocity.

\section{DISCUSSION}

The results from all the experiments demonstrate clearly the effectiveness of motion as a factor in the process of binocular rivalry. The presentation of stimuli that moved symmetrically away from or towards the centers of the displays reduced the likelihood of nystagmic eye movements' influencing the results. 
It has been suggested that for gratings in rivalry the stimulus strength is a function of spatial frequency and apparent contrast. It is evident from our experiments that this index is influenced by movement. This influence could be incorporated into the measure of stimulus strength by the introduction of a movement factor, $\mathbf{M}(\mathrm{v})$, for a stimulus moving with velocity, $v$. That is, stimulus strength $(\mathrm{Lm})$ of a moving stimulus consists of two components: the strength of the stationary stimulus (Ls) and the movement factor $M(v)$, such that:

$$
\mathrm{Lm}=\mathrm{Ls} \cdot \mathrm{M}(\mathrm{v}) .
$$

It should be noted that movement need not necessarily lead to an increase in stimulus strength. For example, rapid motion would lead to a reduction in apparent contrast and, therefore, of stimulus strength. The movement factor is equal to 1 for a static pattern, and it is greater than 1 for only certain velocity ranges. According to this concept of stimulus strength, motion modifies the contrast-based measure.

The spatial characteristics that influenced the stimulus strength of the static patterns were spatial frequency and orientation. The magnitude of the spatial frequency effects was unexpected, particularly in the strength of the lowest frequencies tested. Our initial concern was only with the effects of motion, and the various static spatial frequency conditions were included simply because of the expansion and contraction of the zooming displays in Experiments 1-3. We anticipated that the effect found in Experiment 1 would reflect the characteristics of the contrast sensitivity function for static patterns. The results from Experiment 2, however, suggested that such was not the case. A 1.3-cycles/deg grating was visible for considerably longer than one at 2.2 or $2.6 \mathrm{cycles} / \mathrm{deg}$. In our experiments, we sampled only a small number of spatial frequencies, and therefore the peak of any distribution could have fallen between values we sampled. Moreover, it is difficult to draw firm conclusions on the effects of spatial frequency when square-wave gratings are presented, particularly when the fundamental frequency is lower than the peak of the contrast sensitivity function. Fahle (1982) found that a blurred line was suppressed by a sharply focused one. The blurring was produced by spatial filtering, so that the line was reduced in contrast and the higher spatial frequency components were removed. Our results suggest that the factor influencing the weaker stimulus strength of the blurred line was the reduced contrast rather than the removal of the higher spatial frequencies from the bar.

The study of orientation effects on binocular rivalry has tended to focus on the oblique effect-the difference in visibility between vertical (or horizontal) and oblique gratings. We found in all our experi- ments that a vertical grating was visible for slightly longer than a horizontal one. This applied to both the static and dynamic conditions. In order to examine the possibility that this effect was not an artifact of the display system (e.g., of the raster scan), we conducted a control experiment using the apparatus of Experiments 1-3. Rivalry was measured with vertical and horizontal gratings (both $2.7 \mathrm{cycles} / \mathrm{deg}$ ) presented on monitors that were either vertical or horizontal. Three subjects were tested in 10 sessions for all grating and monitor combinations. There were no differences in the results obtained for the two monitor orientations: the vertical grating was visible for about $13 \%$ longer than the horizontal, a value that corresponds closely to those obtained in Experiments 1 and 2. Fahle (1982) also found a difference in favor of a vertical pattern, and he suggested this could be related to the significance of vertical contours in normal binocular combination. An alternative interpretation draws upon Hollins's (1980) idea of transient involvement in rivalry between static stimuli. If there was a greater incidence of horizontal eye movements during observation of the rivalrous patterns, then this would favor the visibility of the (moving) vertical contours. This possibility is supported by the absence of such orientation effects when the stimuli are retinally stabilized (Wade, 1974, 1975b).

In summary, movement is a potent factor in determining rivalry suppression, but it does not operate in isolation from the spatial characteristics of the rivalrous patterns.

\section{REFERENCES}

BLAKe, R., \& Fox, R. (1974). Binocular rivalry suppression: Insensitive to spatial frequency and orientation change. Vision Research, 14, 687-692.

Blake, R., Westendorf, D. H., \& Overton, R. (1980). What is suppressed during binocular rivalry? Perception, 9, 223-231.

Breese, B.'B. (1899). On inhibition. Psychological Monographs, $3(1), 1-65$.

Duensing, S., \& Miller, B. (1979). The Cheshire cat effect. Perception, 8, 269-273.

Enoksson, P. (1961). A method for investigation of ocular dominance based on optokinetic nystagmus. Acta Ophthalmologica, 39, 115-140.

Enoksson, P. (1963). Binocular rivalry and monocular dominance studied with optokinetic nystagmus. Acta Ophthalmologica, 41, 544-563.

ENOKsson, P. (1968). Studies in optokinetic binocular rivalry with a new device. Acta Ophthalmologica, 46, 71-74.

FAHLE, M. (1982). Binocular rivalry: Suppression depends on orientation and spatial frequency. Vision Research, 22, 787-800.

Fox, R., \& Check, R. (1968). Detection of motion during binocular rivalry suppression. Journal of Experimental Psychology, 78, 388-395.

Fox, R., Todd, S., \& Bettinger, L. A. (1975). Optokinetic nystagmus as an objective indicator of binocular rivalry. Vision Research, 15, 849-853.

Grindley, C., \& Townsend, V. (1965). Binocular masking induced by a moving object. Quarterly Journal of Experimental Psychology, 17, 97-109. 
Grindley, C., \& Townsend, V. (1966). Further experiments on movement masking. Quarterly Journal of Experimental Psychology, 18, 319-326.

Helmholtz, H. L. F. von (1925). Physiological optics (Vol. 3) (J. P. C. Southall, Trans.). New York: Optical Society of America.

Hollins, M. (1980). The effect of contrast on the completeness of binocular rivalry suppression. Perception \& Psychophysics, 27, 550-556.

KitTerle, F. L., \& Thomas, J. (1980). The effects of spatial frequency, orientation, and color upon binocular rivalry and monocular pattern alternation. Bulletin of the Psychonomic Society, 16, 405-407.

LEvelt, W. J. M. (1965). On binocular rivalry. Soesterberg, The Netherlands: Institute for Perception RVO-TNO.

SpringbetT, B. M. (1961). Some stereoscopic phenomena and their implications. British Journal of Psychology, 52, 105-109.

Swanston, M. T., \& Wade, N. J. (1981a). Apparent rotation of a line superimposed upon radially expanding and contracting backgrounds. Perception, 10, 265-271.

Swanston, M. T., \& Wade, N. J. (1981b). Level of response categorization and reporting consistency for rivalry between lines viewed as real images and as afterimages. Perception, 10, 637-643.
Du Tour, E. F. (1760). Discussion d'une question d'optique. Mémoires de mathématique et de physique, 3, 514-530.

WADE, N. J. (1974). The effect of orientation in binocular rivalry of real images and afterimages. Perception \& Psychophysics, $15,227-232$.

WADE, N. J. (1975a). Monocular and binocular rivalry between contours. Perception, 4, 85-95.

WADE, N. J. (1975b). Binocular rivalry between single lines viewed as real images and afterimages. Perception \& Psychophysics, 17, 571-577.

DE WeERT, C. M. M., \& LevelT, W. J. M. (1974). Binocular brightness combinations: Additive and nonadditive aspects. Perception \& Psychophysics, 15, 551-562.

Wheatstone, C. (1838). On some remarkable, and hitherto unobserved, phenomena of binocular vision. Part the first. Philosophical Transactions of the Royal Society, 128, 371-394.

WitTebrood, J. E. M., Wansink, M. G., \& DE Weert, C. M. M. (1981). A versatile colour stimulus generator. Perception, 10, 63-69.

(Manuscript received July 6, 1983; revision accepted for publication October 7,1983 .) 